\title{
Acceptor Substrate Selectivity and Kinetic Mechanism of Bacillus subtilis TagA
}

\author{
Yu-Hui Zhang, Cynthia Ginsberg, Yanqiu Yuan and Suzanne Walker
}

Department of Microbiology and Molecular Genetics, Harvard Medical School, Boston, Massachusetts 02115, and Department of Chemistry and Chemical Biology, Harvard University, Cambridge, Massachusetts 02138

\section{Supporting Information}




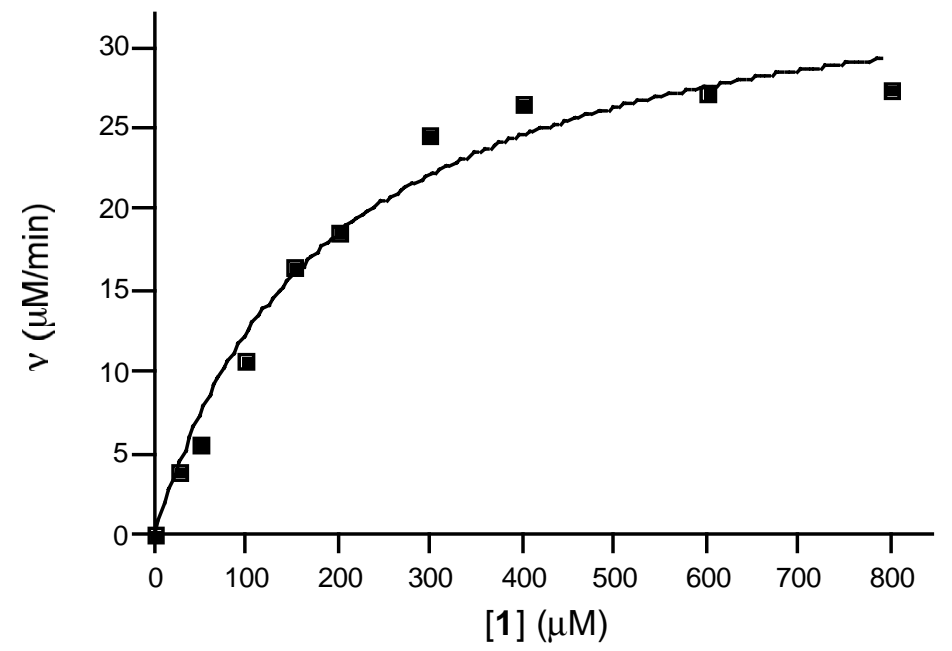

(A)

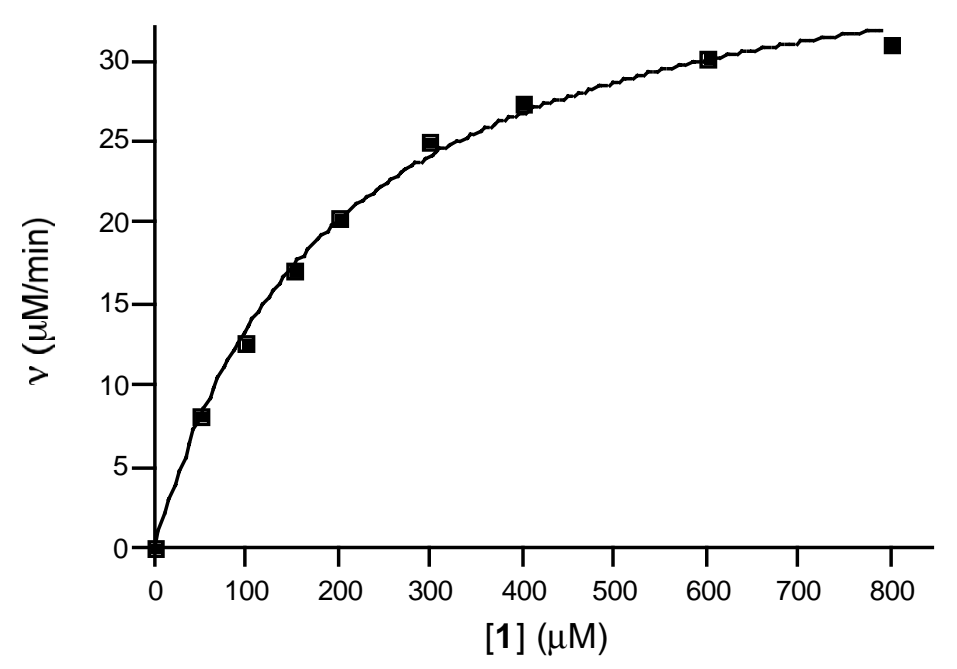

(B)

Figure 1. Michaelis-Menten plots for lipid I analog 1 at $600 \mu \mathrm{M}$ UDP-ManNAc (50 mM Tris, $\mathrm{pH} 7.8,250 \mathrm{mM} \mathrm{NaCl}, 66 \mathrm{nM}$ TagA). Analysis of a single set of reactions was performed using both the HPLC and LC/Ms assays. (A) HPLC assay, $K_{m \text {, app }}=193 \pm 34$ $\mu \mathrm{M}, V_{\max }=36.4 \pm 2.4 \mu \mathrm{M} / \mathrm{min}$. (B) LC/MS assay, $K_{m, a p p}=193 \pm 13 \mu \mathrm{M}, V_{\max }=39.7 \pm 1.0$ $\mu \mathrm{M} / \min$. 


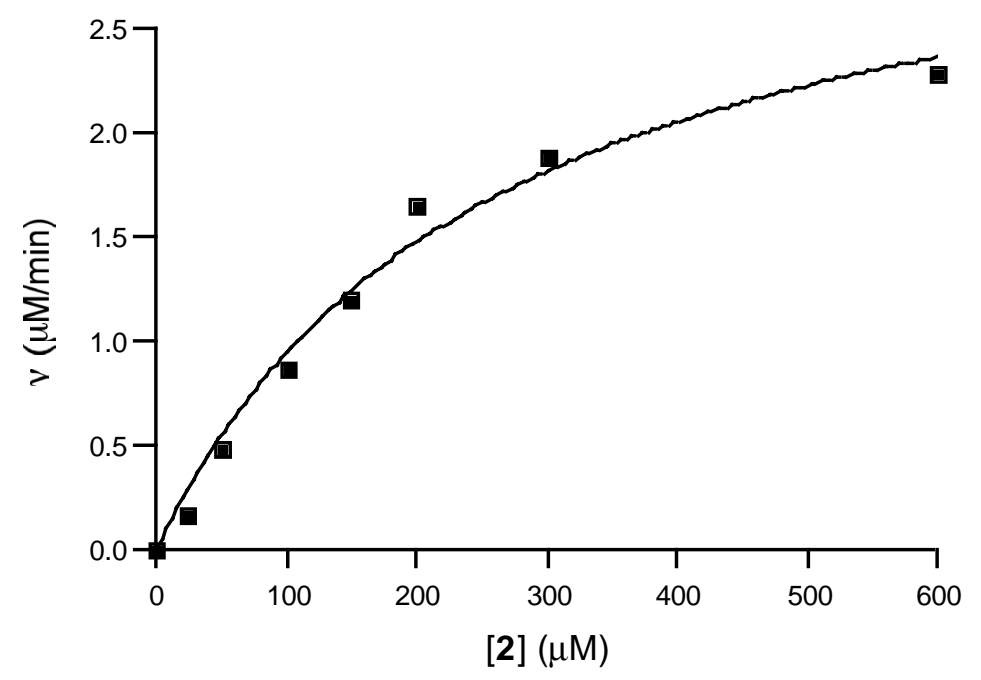

Figure 2. Michaelis-Menten plot for lipid I analog 2 at $600 \mu \mathrm{M}$ UDP-ManNAc $(50 \mathrm{mM}$ Tris, pH 7.8, $250 \mathrm{mM} \mathrm{NaCl}, 66 \mathrm{nM}$ TagA). $K_{m, a p p}=258 \pm 49 \mu \mathrm{M}, V_{\max }=3.4 \pm 0.3$ $\mu \mathrm{M} / \mathrm{min}$.

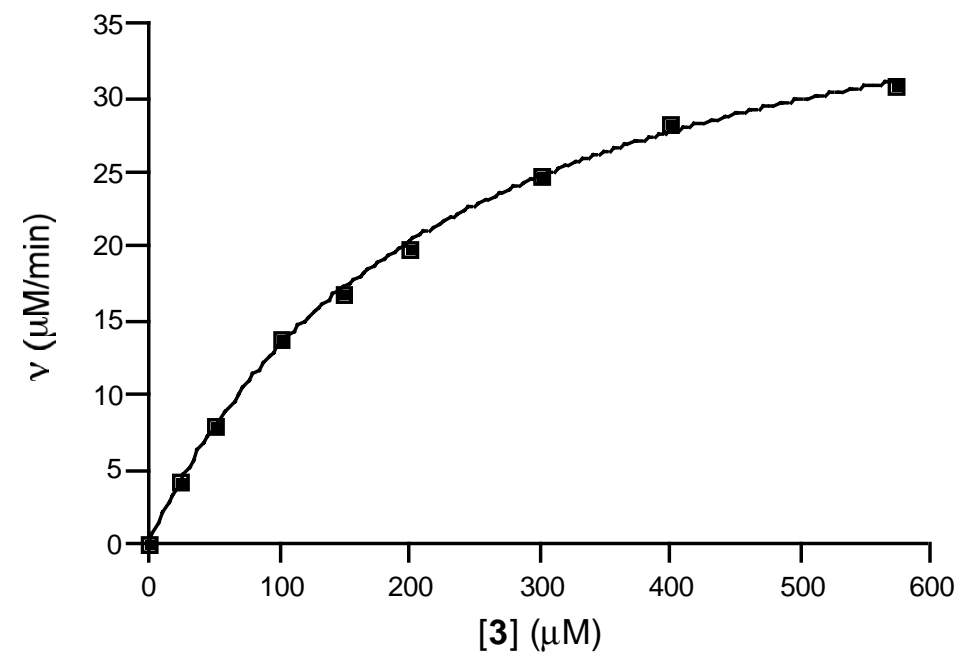

Figure 3. Michaelis-Menten plot for lipid I analog 3 at $600 \mu \mathrm{M}$ UDP-ManNAc $(50 \mathrm{mM}$ Tris, pH 7.8, $250 \mathrm{mM} \mathrm{NaCl}, 52 \mathrm{nM}$ TagA). $K_{m, ~ a p p}=227 \pm 10 \mu \mathrm{M}, V_{\max }=43.4 \pm 0.9$ $\mu \mathrm{M} / \mathrm{min}$. 


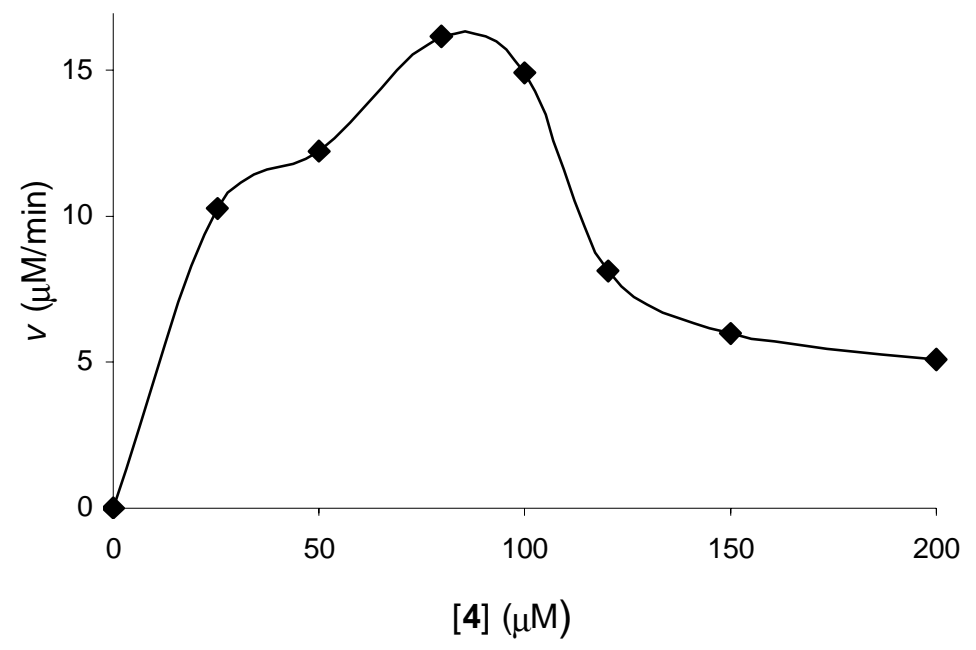

Figure 4. (A) Plot of initial velocity vs. [lipid I analog 4] at $600 \mu \mathrm{M}$ UDP-ManNAc (50 $\mathrm{mM}$ Tris, $\mathrm{pH} 7.8,250 \mathrm{mM} \mathrm{NaCl}, 10 \%$ DMSO, $26 \mathrm{nM}$ TagA). $K_{m, a p p}=30 \mu \mathrm{M}, V_{\max }=16$ $\mu \mathrm{M} /$ min (crude estimates based on the initial rate data). The data cannot be fit to the Michaelis-Menten equation because of the substrate inhibition observed above $100 \mu \mathrm{M}$ and the apparent $K_{m}$ is thus estimated as the substrate concentration at $1 / 2 \times$ highest observed rate. 


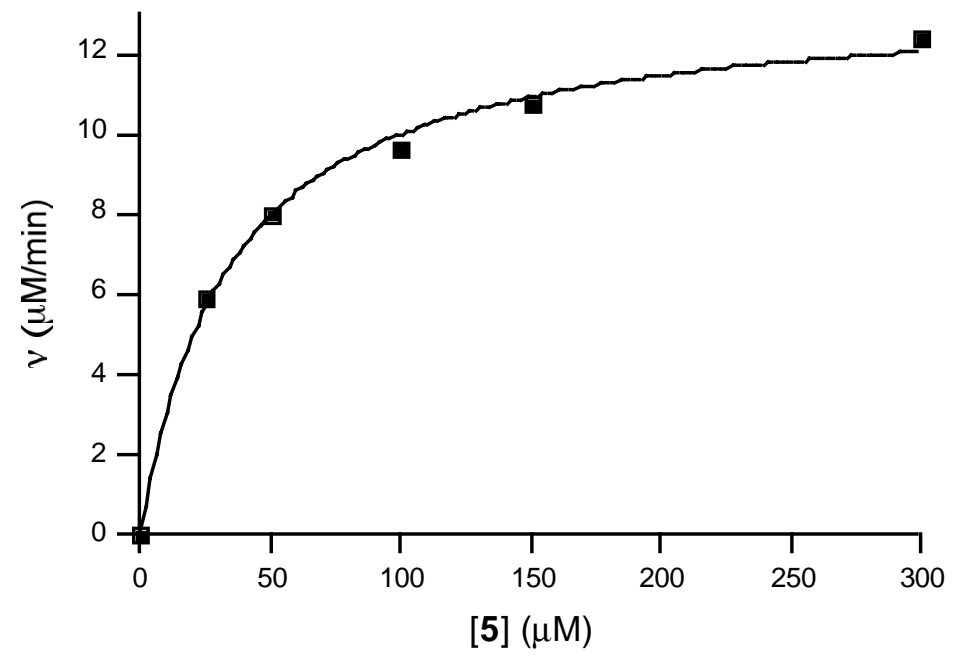

(A)

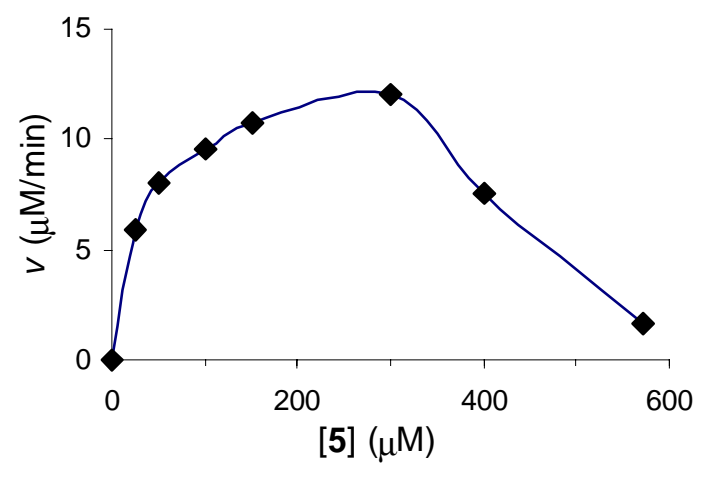

(B)

Figure 5. (A) Michaelis-Menten plot for lipid I analog 5 at $600 \mu \mathrm{M}$ UDP-ManNAc (50 mM Tris, $\mathrm{pH} 7.8,250 \mathrm{mM} \mathrm{NaCl}, 10 \%$ DMSO, $26 \mathrm{nM}$ TagA). $K_{m, \text { app }}=34.5 \pm 3.6 \mu \mathrm{M}, V_{\max }$ $=13.44 \pm 0.38 \mu \mathrm{M} / \mathrm{min}$. (B) Plot of initial velocity vs. [5], showing substrate inhibition at concentrations above $300 \mu \mathrm{M}$. 


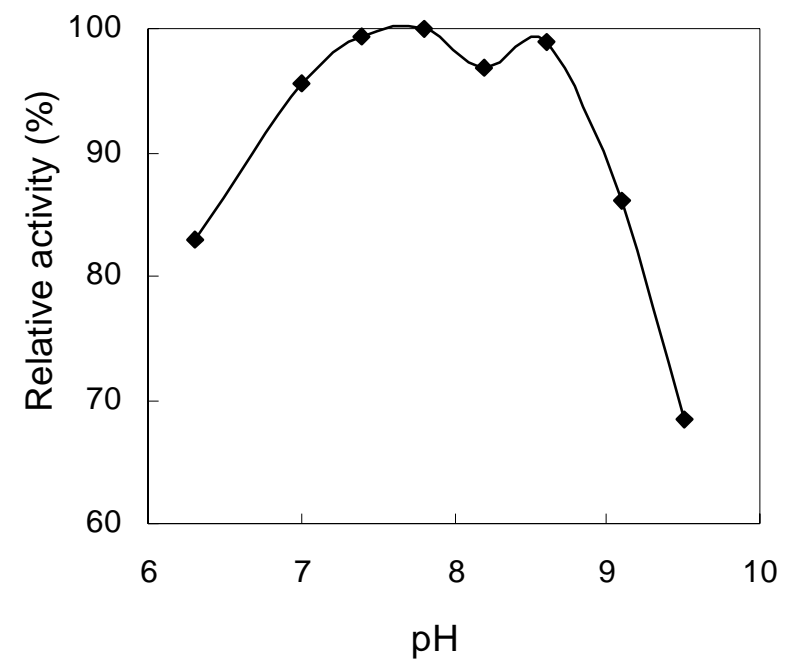

Figure 6. The effect of $\mathrm{pH}$ on TagA activity. Assays were carried out at $100 \mu \mathrm{M}$ UDP-ManNAc, $100 \mu \mathrm{M}$ lipid I analog 1 and $100 \mathrm{nM}$ TagA (50 mM Bis-Tris propane, 250 $\mathrm{mM} \mathrm{NaCl})$.

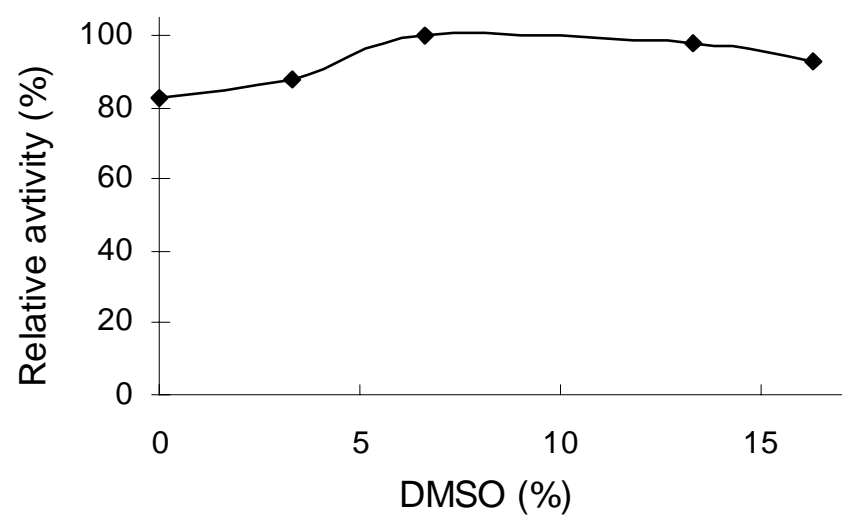

Figure 7. The effect of DMSO on TagA activity. Assays were carried out at $100 \mu \mathrm{M}$ UDP-ManNAc, $100 \mu \mathrm{M}$ lipid I analog 1 and $52 \mathrm{nM}$ TagA (50 mM Tris-HCl, pH 7.8, 250 $\mathrm{mM} \mathrm{NaCl})$. 
Table 1. Expected product inhibition patterns for selected ordered and random Bi Bi kinetic mechanisms (1).

\begin{tabular}{|c|c|c|c|c|c|}
\hline \multirow[b]{2}{*}{ mechanism } & \multirow{2}{*}{$\begin{array}{l}\text { product } \\
\text { inhibitor }\end{array}$} & \multicolumn{2}{|c|}{ varying $\mathrm{A}$} & \multicolumn{2}{|c|}{ varying $B$} \\
\hline & & unsat. B & sat.B & unsat.A & sat. A \\
\hline rapid equi. random $\mathrm{Bi} \mathrm{Bi}$ & $\mathrm{P}$ & $\mathrm{C}$ & NI & $\mathrm{C}$ & NI \\
\hline with dead-end EBQ & Q & $\mathrm{C}$ & $\mathrm{C}$ & $\mathrm{NC}$ & NI \\
\hline rapid equi. random $\mathrm{Bi} \mathrm{Bi}$ & $\mathrm{P}$ & NC & NI & $\mathrm{C}$ & $\mathrm{C}$ \\
\hline with dead-end EAP and EBQ & Q & $\mathrm{C}$ & $\mathrm{C}$ & $\mathrm{NC}$ & NI \\
\hline steady-state & $\mathrm{P}$ & $\mathrm{NC}$ & $\mathrm{UC}$ & $\mathrm{NC}$ & $\mathrm{NC}$ \\
\hline \multirow[t]{2}{*}{ ordered $\mathrm{Bi} \mathrm{Bi}$} & Q & $\mathrm{C}$ & $\mathrm{C}$ & $\mathrm{NC}$ & NI \\
\hline & $\mathrm{P}$ & $\mathrm{NC}$ & NI & $\mathrm{C}$ & $\mathrm{C}$ \\
\hline Theorell-Chance & Q & $\mathrm{C}$ & $\mathrm{C}$ & $\mathrm{NC}$ & NI \\
\hline
\end{tabular}

C, competitive inhibition; $\mathrm{NC}$, noncompetitive inhibition; UC, uncompetitive inhibition;

NI, no inhibition

\section{References}

1. Leskovac V, Ed. (2003) Comprehensive Enzyme Kinetics pp 180, Kluwer Academic Press, Seacaucus, NJ. 